\title{
SRS and XPM Impairments on Different Optical Fibers
}

\author{
Ravi Gupta Manisha \\ Department of ECE, Government Engineering College of Bharatpur, Bharatpur, India \\ Email: raviguptabtp@gmail.com
}

Received 18 February 2016; accepted 24 April 2016; published 27 April 2016

Copyright ( 2016 by author and Scientific Research Publishing Inc.

This work is licensed under the Creative Commons Attribution International License (CC BY). http://creativecommons.org/licenses/by/4.0/

cC) (i) Open Access

\begin{abstract}
In this paper, the analysis of crosstalk induced by stimulated Raman scattering has been discussed. The expression for SRS induced crosstalk has been observed at different fiber types, such as single mode fiber, DCF, NZDF and NZDSF with the variation of modulation frequency, transmission length and the input power. It has been observed that as an increase in the modulation frequency, the crosstalk decreases.
\end{abstract}

\section{Keywords}

SRS, DCF, NZDF, NZDSF, Modulation Frequency, Transmission Length

\section{Introduction}

An optical communication system is used to transfer the information from transmitter to receiver, which is separated by a few kilometres distances. Message is carried by an electromagnetic carrier wave whose frequency can vary from a few megahertz to several hundred terahertz [1]. Virtually there are only two windows used for broadband communications. The first window is between $100 \mathrm{KHz}$ and $300 \mathrm{GHz}$, and the second window from $30 \mathrm{THz}$ to $300 \mathrm{THz}$. Now the demand for bandwidth is increasing enormously, so the second window is used which is optical and has a capacity of $100 \mathrm{~Tb} / \mathrm{s}$ and beyond. The initial development of optical fiber was for long haul or submarine transmission, but now everywhere optical fibers are used [2]. Year after year, there is development in the optical fiber that needs some changes which help to enhance the system performance further. Optical networks are divided into optical active network (OAN) and optical passive network (OPN) [3]. Fiber-to-the-Home (FTTH) is the main focus of telecommunication industry. Direct fiber connection has surplus advantage of large bandwidth and low maintenance cost. However, FTTH can only remain competitive with the help of a passive optical network. SCM is a potential solution for transmission in OPNs [4]. The combination of SCM and WDM is a feasible method to further increase the transmission capacity in OPNs [5]. SCM-WDM systems, however, are affected from non-linear effects in fiber. Such non-linearities cause crosstalk among sub- 
scribers on different wavelengths. In a dispersive fiber, the dominant fiber nonlinearity that causes crosstalk is cross-phase modulation (XPM). Fiber nonlinearities such as stimulated Raman scattering (SRS) and cross phase modulation (XPM) may generate significant amount of nonlinear crosstalk among adjacent SCM channels since they are very closely spaced [2] [6]. Crosstalk, mainly due to SRS, SBS and XPM, occurs due to nonlinearities of the fiber SRS effect, is more dominant for the frequencies which are adjoining to the transmitted ones [7] [8]. The crosstalk levels obtained to date indicated that crosstalk in SCM-WDM systems could easily reach intolerable levels even with two wavelengths.

\section{Crosstalk}

There are two types of crosstalk in optical fiber: Linear and Non Linear. In this paper, we have focussed on Non Linear crosstalk i.e. SRS and XPM.

\subsection{Crosstalk Induced by SRS}

In this type of analysis, two optical waves of different modulation index, amplitudes and phases have been taken into account [9] [10].

Crosstalk is determined with the help of following coupled equation leading phase modulation given by

$$
\begin{aligned}
& \frac{\partial S_{1}}{\partial z}+1 / V_{g 1} \frac{\partial S_{1}}{\partial t}=\left(g S_{2}-\alpha\right) S_{1} \\
& \frac{\partial S_{2}}{\partial z}+1 / V_{g 2} \frac{\partial S_{2}}{\partial t}=\left(g S_{1}-\alpha\right) S_{2} .
\end{aligned}
$$

where $V_{g_{1}}$ is the group velocity for the transmitted signal at $\lambda_{1} ; V_{g_{2}}$ is the group velocity for the transmitted signal at $\lambda_{2} ; \alpha$ is the fiber loss coefficient; $g$ is the standard coefficient divided by the fiber effective area $\left(g=g_{R} / A_{e f f}\right)$.

$$
S_{1}(z, t)=S_{1}\left(0, \tau_{1}\right) e^{-\alpha z}
$$

We first solve for $S_{1}$ in Equation (1) by neglecting. We then substitute $S_{1}$ into (2) to solve for $S_{2}$ to obtain

$$
\begin{aligned}
& S_{0} e^{-\alpha z}\left[1-g S_{0}\right]\left[\frac{1-e^{-\alpha z}}{\alpha}\right]-g m S_{0} \frac{\sqrt{1+e^{-2 \alpha z}-2 e^{-\alpha z} \cos \left(\omega d_{12} z\right)}}{\sqrt{\alpha^{2}+\left(\omega d_{12}\right)^{2}}} \cos \left(\omega \tau_{2}+\Theta_{S R S}\right) \frac{\partial S_{2}}{\partial z}+1 / V_{g 2} \frac{\partial S_{2}}{\partial t} \\
& =\left(g S_{1}-\alpha\right) S_{2}
\end{aligned}
$$

where

$$
\Theta_{S R S}=\tan ^{-1}\left(\frac{-\omega d_{12}}{-\alpha}\right)+\tan ^{-1} e^{-\frac{e^{-\alpha z} \sin \left(\omega d_{12} z\right)}{-\alpha z}\left(\omega d_{12} z\right)-1}
$$

Hence, $g=\frac{g_{R}}{A_{\text {eff }}}$.

In Equation (4), the first term represent to the carrier power after fiber loss. The second term represent to the interaction between the optical carriers, this result in optical dc power gain or loss. The third term is the crosstalk as the result of modulation depletion through SRS interaction between pump channel optical carrier and signal channel subcarrier. The crosstalk affected by the subcarrier due to SRS is (5).

$$
\text { Crosstalk, (SRS) [9] }=\left|g S_{0} \frac{\sqrt{1+e^{-2 \alpha z}-2 e^{-\alpha z} \cos \left(\omega d_{12} z\right)}}{A_{e f f} \sqrt{\alpha^{2}+\left(\omega d_{12}\right)^{2}}}\right|^{2}
$$

\subsection{Crosstalk Produced by XPM}

The 3rd OD cross phase modulation Figure 1 is given by

$$
-S_{2}\left(0, \tau_{2}\right) e^{-\alpha z} \beta_{3} \frac{\partial \emptyset}{\partial \tau_{2}} \frac{\partial^{2} \emptyset}{\partial \tau_{2}^{2}} .
$$




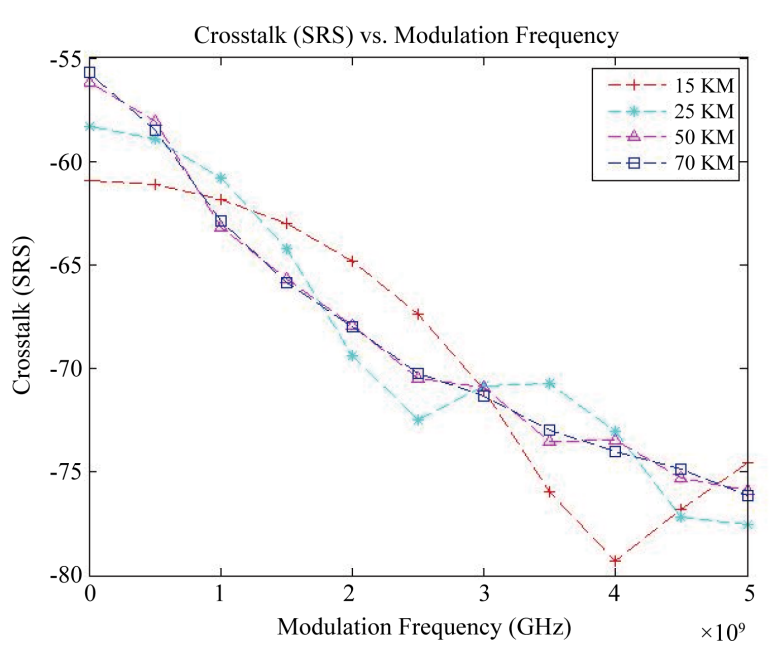

(a)

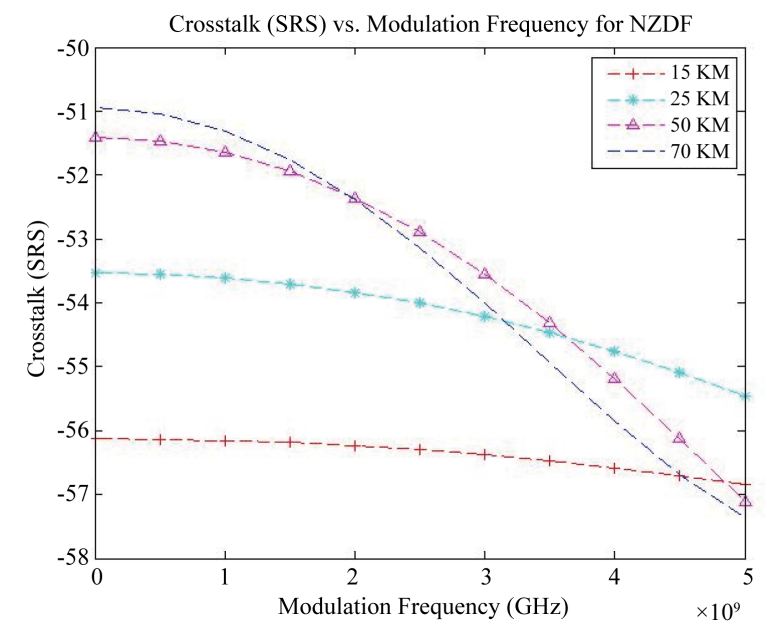

(c)

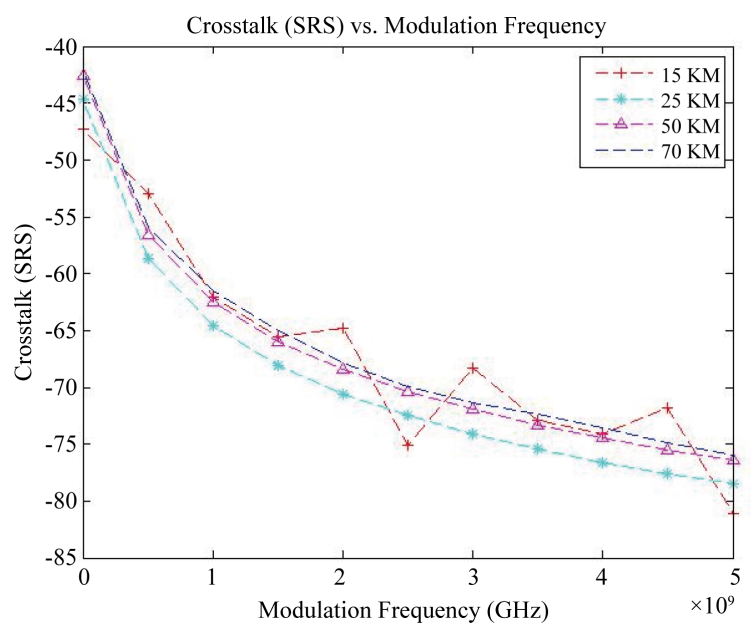

(b)

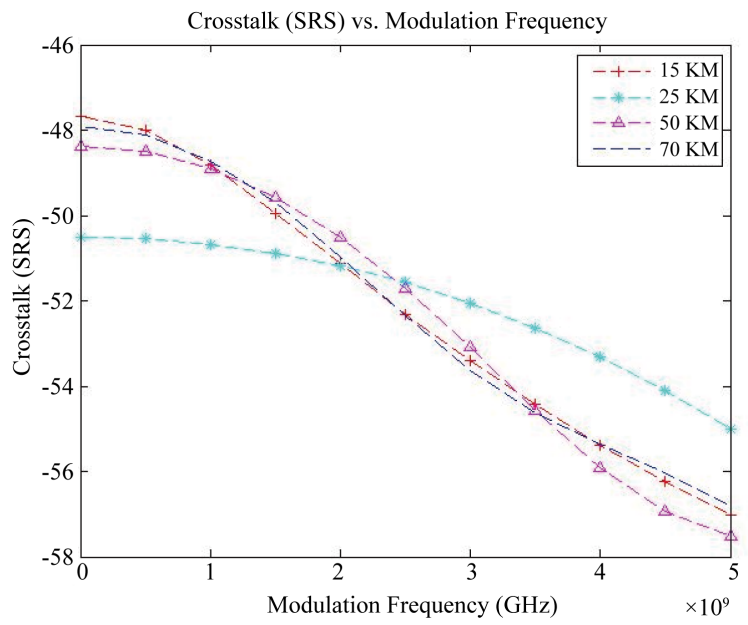

(d)

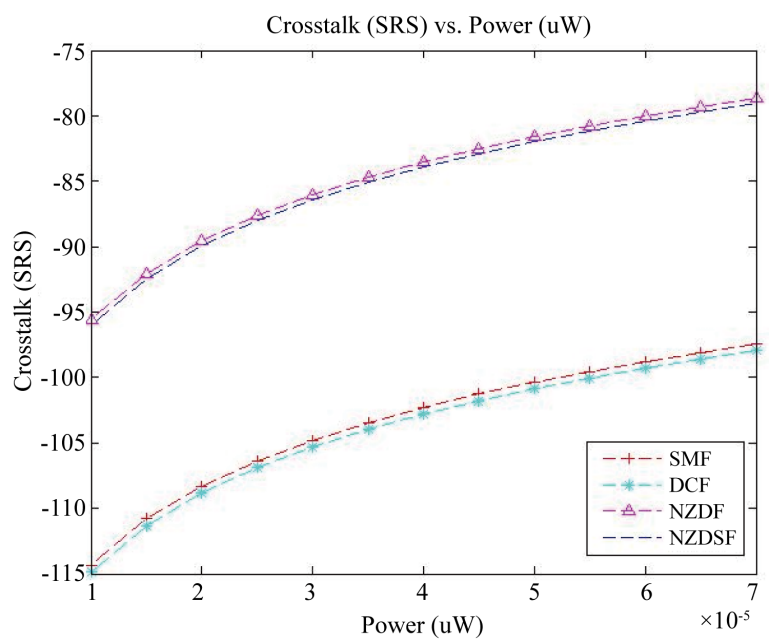

(e)

Figure 1. (a) Crosstalk vs. Modulation Frequency for SMF; (b) Crosstalk vs. Modulation Frequency for DCF; (c) Crosstalk vs. Modulation Frequency for NZDF; (d) Crosstalk vs. Modulation Frequency for NZDSF; (e) Crosstalk vs. Power for different optical fibers. 
$\beta_{3}=3 \mathrm{OD}$ parameter.

$$
\frac{\partial^{3} \beta}{\partial \omega^{3}}=\frac{1}{(2 \pi c)^{2}}\left[\lambda^{2} D_{1}+2 \lambda D\right]
$$

Crosstalk (XPM) 3OD =

$$
-\frac{2 m \beta_{s} \gamma^{2} S_{c} \omega^{3}}{\left(\alpha-j \omega d_{12}\right)^{3}}\left\{\left(3+2 \alpha l+4 e^{-\alpha l} \cos \left(\omega d_{12} l\right)-e^{-2 \alpha l} \cos \left(2 \omega d_{12} l\right)+j 4 e^{-\alpha l} \sin \left(\omega d_{12} l\right)-e^{-2 \alpha l} \sin \left(2 \omega d_{12} l\right)-2 \omega d_{12} l\right)\right\}
$$

\section{Result Analysis}

Here, the results show SRS induced crosstalk using different fiber parameters variation in modulation frequency in range of $0-5 \mathrm{GHz}$, optical power and transmission length.

\subsection{Crosstalk Produced by SRS}

The graph between SRS induced crosstalk with modulation frequency with varied fiber parameters shown in Figure 2, Table 1. There are different type of fibers are used such as SMF, DCF, NZDF and NZDSF and

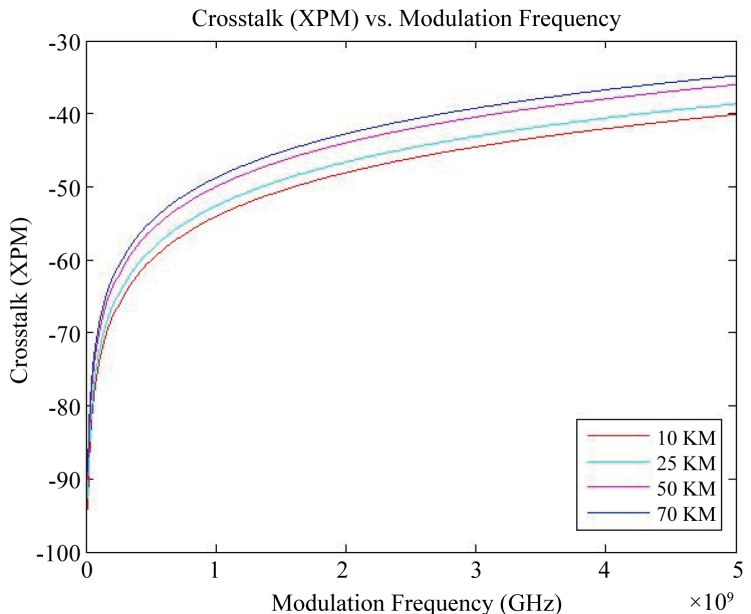

(a)

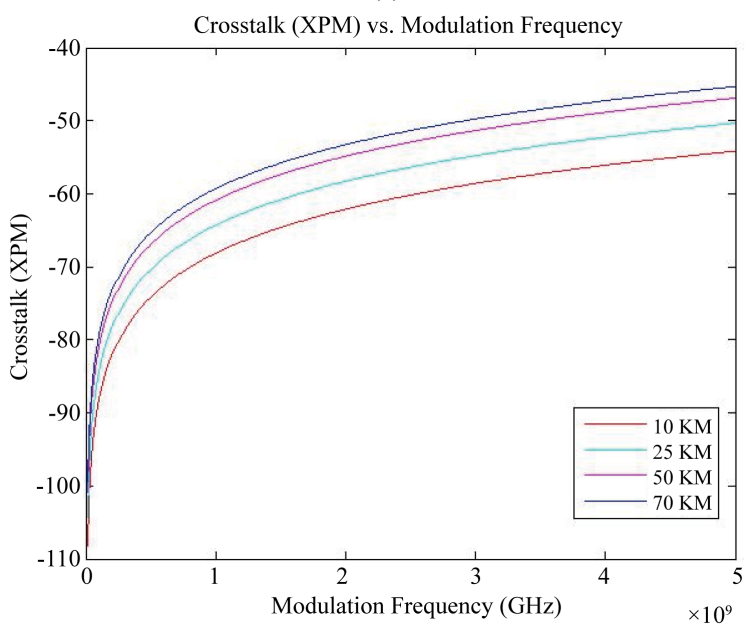

(c)

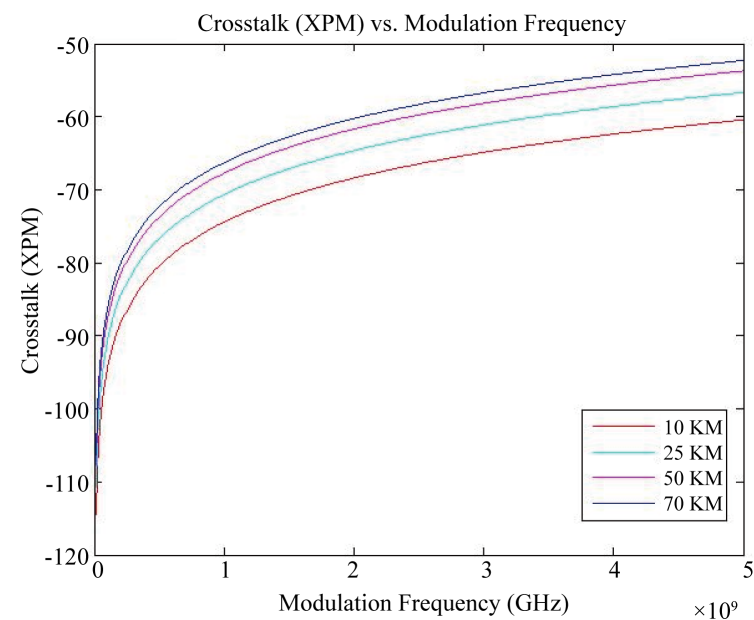

(b)

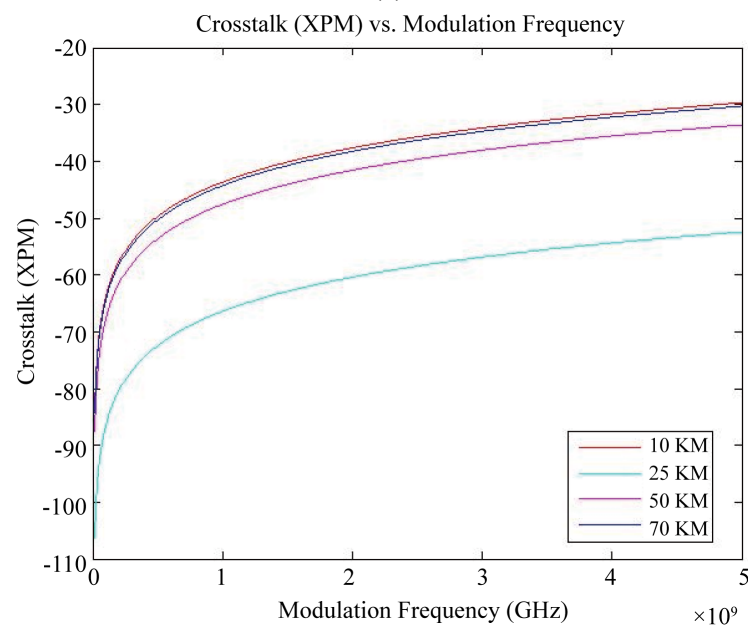

(d)

Figure 2. Crosstalk vs. Modulation Frequency: (a) SMF; (b) DCF; (c) NZDF; (d) NZDSF. It shows the graph between SRS and XPM induced crosstalk with transmission length with varied fiber parameters. 
Table 1. Parametric characteristics of different fiber types [11].

\begin{tabular}{ccccc}
\hline Fiber type & $\mathrm{A}_{\text {eff }}\left(\mu \mathrm{m}^{2}\right)$ & $\mathrm{D}_{\mathrm{c}}$ & $\alpha$ & $\mathrm{g}(\mathrm{Km} / \mathrm{W})$ \\
\hline $\mathrm{SMF}$ & 80 & 17 & 0.2 & 2.5 \\
$\mathrm{DCF}$ & 20 & -80 & 0.29 & 3 \\
$\mathrm{NZDF}$ & 72 & -3 & 0.23 & 3.9 \\
NZDSF & 50 & 4.5 & 0.25 & 3.84 \\
\hline
\end{tabular}

modulation frequency is varied from 0 to $5 \mathrm{GHz}$. It has been observed that as the modulation frequency increases the SRS induced crosstalk decreases. Moreover the decrease in crosstalk depends on the fiber type used $[11]$.

\section{Conclusion}

This paper shows SRS induced crosstalk in an optical fiber communication transmission system for different types of optical fibers, modulation frequency and optical power. It also shows in the results that the crosstalk due to SRS increases with increase length of transmission and decreases with the increase in modulation frequency \& optical power. By the above results, the optical fiber communication transmission system can reduce the crosstalk by taking appropriate transmission length and using type of fiber. Initially the crosstalk remains high and then it decreases with increase in modulation frequency. Out of four different types of fiber standards, SMF and DCF have minimum SRS and XPM as ( -61 to -74$),(-46$ to -79$)$ in comparison with [9] and NZDSF has the maximum crosstalk of $(-47$ to -57$)$. Result also shows that with increase of transmission length, crosstalk produced by SRS also is increased and decreased with the increase in modulation frequency and power. With increase in modulation frequency and transmission length, crosstalk produced by XPM at higher order dispersion is increased. As shown in above results, the optical fiber communication transmission system may improve in a given range of modulation frequency, optical power and length of transmission by selecting the minimum value of crosstalk.

\section{References}

[1] Ho, K.P. and Kahn, J.M. (2001) Method for Cross Talk Measurement and Reduction in Dense WDM.

[2] Hui, R., Zhu, B., Huang, R., Allen, C., Demarest, K. and Roberts, D. (2001) 10Gb/s SCM Systems Using Optical Single Side Band Modulation. Paper MM4, OFC'2001, Anaheim.

[3] Gros, R. and Olshansky, R. (1990) Multichannel Coherent FSK Experiments Using Subcarrier Multiplexing Techniques. IEEE Journal of Light wave Technology, 8, 406-415. http://dx.doi.org/10.1109/50.50737

[4] Ho, K.P. and Kahn, J.M. (1996) Method for Cross Talk Measurement and Reduction in Dense WDM Systems. Journal of Light wave Technology, 14, 1127-1135. http://dx.doi.org/10.1109/50.511614

[5] Goeger, G., Wrage, M. and Fischler, W. (2004) Cross-Phase Modulation in Multispan WDM Systems with Arbitrary Modulation Formats. IEEE Photonics Technology Letters, 16, 1858-1860. http://dx.doi.org/10.1109/LPT.2004.830519

[6] Lu, H.-H., Wang, W.J. and Tsai, W.-S. (2004) CSO/CTB Performances Improvement in a Bi-Directional DWDM CATV System. IEEE Transactions on Broadcasting, 50, 377-381. http://dx.doi.org/10.1109/TBC.2004.837880

[7] Maiman, T.H. (1960) Stimulated Optical Radiation in Ruby.

[8] Karfaa, Y.M., Ismail, M., Abbou, F.M. and Shaari, A.S. (2008) Theoretical Evaluation of Non-Linear Effects on Optical WDM Networks with Various Fiber Types. IIUM Engineering Journal, 9, 53-56.

[9] Kumar, N., Sharma, A.K. and Kapoor, V. (2011) Performance Evaluation of SCM-WDM Communication in the Presence of SRS Induced Crosstalk for Different Fiber Types. Elsevier OPTIK, 122, 1862-1864. http://dx.doi.org/10.1016/j.ijleo.2010.12.028

[10] Kapron, F.P., Keck, D.B. and Maurer, R.D. (1970) Radiation Losses in Glass Optical Waveguides. Applied Physics Letters, 17, 423-425. http://dx.doi.org/10.1063/1.1653255

[11] Djupskobacka, A., Jacobson, G. and Tromborg, B. (2000) Dynamic Stimulated Scattering Analysis. Journal of Lightwave Technology, 18, 416-424. http://dx.doi.org/10.1109/50.827515 